Joan E. Hart*, Mathematics Department, The University of Dayton, Dayton, OH 45469-2316, USA. e-mail: jhart@udayton.edu

Kenneth Kunen*, Mathematics Department, University of Wisconsin, Madison, WI 53706-1388, USA. e-mail: kunen@math.wisc.edu

\title{
ORTHOGONAL CONTINUOUS FUNCTIONS
}

\begin{abstract}
We consider the question of whether there is an orthonormal basis for $L^{2}$ consisting of continuous functions.
\end{abstract}

\section{Introduction}

In elementary analysis, the typical orthonormal bases for $L^{2}[0,1]$ (trig functions, orthogonal polynomials, etc.) frequently consist of continuous functions. It is natural to ask whether such orthonormal bases must exist if $[0,1]$ is replaced by a more general space and measure. One commonly studied generalization of $[0,1]$ is:

Definition 1.1. $(X, \nu)$ is a nice measure space iff $X$ is a compact Hausdorff space and $\nu$ is a regular Borel probability measure on $X$ which is strictly positive (i.e., all non-empty open sets have positive measure).

The assumption that $\nu$ is strictly positive is mainly for notational convenience. In general, one can simply delete the union of all open null sets to obtain a strictly positive measure.

Since $\nu$ is strictly positive, distinct elements of $C(X)$ do not become equivalent in $L^{2}$, so we may regard $C(X)$ as contained in $L^{2}(X, \nu)$. There are then two well-known situations where there is an $\mathcal{F} \subseteq C(X)$ which forms an orthonormal basis for $L^{2}(X, \nu)$. The first is whenever $L^{2}(X, \nu)$ is separable (by Gram-Schmidt). The second is when $X$ is a compact group and $\nu$ is Haar measure (by the Peter-Weil Theorem; see, e.g., Folland [1]). However, there

Key Words: function space, orthonormal basis

Mathematical Reviews subject classification: 28C15, 46E20

Received by the editors May 17, 1999

*The authors were supported by NSF Grant DMS-9704520. 
need not be such an $\mathcal{F}$ in general, since Theorem 3.6 yields an example where $L^{2}(X, \nu)$ is not separable but every orthogonal family from $C(X)$ is countable.

In the example of Theorem 3.6, $X$ is actually a topological group, since it is a product of two-element spaces, and $\nu$ looks a bit like the product measure, which in this case would be Haar measure. Nevertheless, by Theorem 2.5, no such $\nu$ can be absolutely continuous with respect to Haar measure.

The proof for the specific example of Theorem 3.6 works equally well whether one considers the scalar field to be $\mathbb{R}$ or $\mathbb{C}$. However, if one starts with an arbitrary nice $(X, \nu)$, it is reasonable to ask whether the properties discussed here can depend on the scalar field. They do not, as we show in Corollary 2.2. Of course, any orthogonal family of real-valued functions remains orthogonal when viewed as a subfamily of $L^{2}(X, \nu, \mathbb{C})$, but Corollary 2.2 explains how to replace orthogonal complex-valued functions by real-valued ones. The familiar method from Fourier series replaces $\varphi$ and $\bar{\varphi}$ by $(\varphi+\bar{\varphi}) / \sqrt{2}$ and $(\varphi-\bar{\varphi}) /(i \sqrt{2})$, but this requires assuming that $\varphi \in \mathcal{F} \Longleftrightarrow \bar{\varphi} \in \mathcal{F}$.

One might study the following property of $X$ : For every finite regular Borel measure $\nu$ on $X$, there is an $\mathcal{F} \subseteq C(X)$ which forms an orthonormal basis for $L^{2}(X, \nu)$. We do not know whether this is equivalent to some interesting topological property of $X$. Note that every compact F-space and every compact metric space has this property.

\section{Basics}

Throughout, when discussing $C(X)$ and $L^{2}(X, \nu)$ and general Hilbert spaces, we always presume that the scalar field is the complex numbers. We shall show that we can convert a family of orthogonal continuous functions to a family of real-valued orthogonal continuous functions with the same span. To do this, we use the following lemma about Hilbert spaces, which gives us a uniform way to transform an "almost orthogonal" family to an orthogonal one:

Lemma 2.1. Suppose that $\mathcal{H}$ is a Hilbert space and $\mathcal{E} \subseteq \mathcal{H}$ is such that the closed linear span of $\mathcal{E}$ is $\mathcal{H}$ and $\{g \in \mathcal{E}:(g, f) \neq 0\}$ is countable for all $f \in \mathcal{E}$. Then there is an orthonormal basis $\mathcal{F}$ for $\mathcal{H}$ such that each element of $\mathcal{F}$ is a finite linear combination of elements of $\mathcal{E}$. Furthermore, if for all $g, f \in \mathcal{E}$ $(g, f)$ is real, then the coefficients in these linear combinations are real.

Proof. On $\mathcal{E}$, let $\sim$ be the smallest equivalence relation such that $g \sim f$ whenever $(g, f) \neq 0$. Let $\mathcal{E}_{j}$, for $j \in J$, list all the $\sim$ equivalence classes. Then the $\mathcal{E}_{j}$ are all countable, and are pairwise orthogonal. For each $j$, apply Gram-Schmidt to obtain an orthonormal family $\mathcal{F}_{j}$ with the same linear span, 
such that the elements of $\mathcal{F}_{j}$ are linear combinations of elements of $\mathcal{E}_{j}$. Then, let $\mathcal{F}=\bigcup_{j} \mathcal{E}_{j}$.

Corollary 2.2. Suppose that $(X, \nu)$ is a nice measure space and $\mathcal{G} \subseteq C(X)$ is an orthonormal family. Then there is an orthonormal family $\mathcal{F} \subseteq C(X)$, consisting of real-valued functions, such that the closed linear span of $\mathcal{F}$ contains the closed linear span of $\mathcal{G}$.

Proof. As usual, write each $G \in \mathcal{G}$ as $G=\Re(G)+i \Im(G)$, where $\Re(G)$ and $\Im(G)$ are real-valued functions. Let $\mathcal{E}=\{\Re(G): G \in \mathcal{G}\} \cup\{\Im(G): G \in \mathcal{G}\}$. Then the closed linear span $\mathcal{H}$ of $\mathcal{E}$ contains $\mathcal{G}$, so Lemma 2.1 will apply if we can verify that $\{g \in \mathcal{E}:(g, f) \neq 0\}$, for any $f \in \mathcal{E}$, is countable. To see this, apply Bessel's inequality: $\sum_{G \in \mathcal{G}}|(G, f)|^{2} \leq\|f\|^{2}$. Since $f$ is real-valued, $|(G, f)|^{2}=(\Re(G), f)^{2}+(\Im(G), f)^{2}$, so that $(\Re(G), f)=(\Im(G), f)=0$ for all but countably many $G \in \mathcal{G}$.

In particular, if $\mathcal{G}$ is an orthonormal basis, we may replace $\mathcal{G}$ by a realvalued orthonormal basis $\mathcal{F}$. Or, if $\mathcal{G}$ is an uncountable orthonormal family, then $\mathcal{F}$ will be a real-valued uncountable orthonormal family. So, the properties of $(X, \nu)$ considered in this paper do not depend on the scalar field.

The next definition and lemma give us a way of ensuring that there are no uncountable orthonormal families within $C(X)$.

Definition 2.3. We say $\mathcal{F} \subseteq C(X)$ is maximal orthogonal iff $\mathcal{F}$ is orthogonal in $L^{2}(X, \nu)$ and there is no orthogonal $\mathcal{G}$ with $\mathcal{F} \varsubsetneqq \mathcal{G} \subseteq C(X)$.

Observe that even in $L^{2}([0,1])$, a maximal orthogonal $\mathcal{F} \subseteq C([0,1])$ need not be an orthogonal basis for $L^{2}([0,1])$. For example, its closed linear span may be the orthogonal complement of a step function (since the continuous functions are dense in the orthogonal complement of any step function). Nevertheless:

Lemma 2.4. Suppose $(X, \nu)$ is a nice measure space, and assume that there is a maximal orthogonal $\mathcal{F} \subseteq C(X)$ which is countable. Then every orthogonal $\mathcal{G} \subseteq C(X)$ is countable.

Proof. Let $\mathcal{F}$ and $\mathcal{G}$ be any two orthogonal families contained in $C(X)$. For each fixed $f \in \mathcal{F}$, Bessel's Inequality implies that $g \perp f$ for all but countably many $g \in \mathcal{G}$. If $\mathcal{F}$ is maximal then each element of $\mathcal{G}$ fails to be orthogonal to some $f \in \mathcal{F}$; i.e.,

$$
\mathcal{G}=\bigcup_{f \in \mathcal{F}}\{g \in \mathcal{G}:(g, f) \neq 0\}
$$

Thus, if $\mathcal{F}$ is also countable, then so is $\mathcal{G}$. 
Now, the existence of an uncountable orthogonal family contained in $C(X)$ depends on $\nu$, not just $X$, as the example in Section 3 shows. Nevertheless:

Theorem 2.5. Suppose that $(X, \nu)$ and $(X, \mu)$ are nice measure spaces with $\mu \ll \nu$. Suppose that $\mathcal{G} \subseteq C(X)$ is an orthonormal basis for $L^{2}(X, \nu)$. Then there is an $\mathcal{F} \subseteq C(X)$ which is an orthonormal basis for $L^{2}(X, \mu)$.

Proof. Fix a Baire-measurable $\varphi: X \rightarrow[0, \infty)$ such that for all Borel sets $E$, we have $\mu(E)=\int_{E} \varphi(x) d \nu(x)$ (the Radon-Nikodym Theorem guarantees such a $\varphi$ exists). Then $\int \varphi d \nu=1$. If $\varphi$ is bounded, then the closed linear span of $\mathcal{G}$ in $L^{2}(X, \mu)$ is all of $L^{2}(X, \mu)$, and the result follows directly by Lemma 2.1. When $\varphi$ is not bounded, the result still follows by Lemma 2.1, but applied to a different family $\mathcal{E}$ one obtains as follows.

Choose closed $G_{\delta}$ sets $K_{0} \subseteq K_{1} \subseteq K_{2} \subseteq \cdots$ such that $\varphi(x) \leq n$ for all $x \in K_{n}$ and $\nu\left(X \backslash \bigcup_{n} K_{n}\right)=0$. For each $n$, choose $\psi_{n} \in C(X,[0,1])$ such that $K_{n}=\psi_{n}^{-1}\{1\}$, and note that the sequence of functions $\left(\psi_{n}\right)^{m}$ converges pointwise to $\chi_{K_{n}}$ as $m \rightarrow \infty$.

Let $\mathcal{E}$ be the set of all functions of the form $g \cdot\left(\psi_{n}\right)^{m}$, where $g \in \mathcal{G}$ and $m, n \in \mathbb{N}$. Then $\mathcal{E} \subseteq C(X) \subseteq L^{2}(X, \mu)$. Let $\mathcal{H}$ be the closed linear span of $\mathcal{E}$ in $L^{2}(X, \mu)$. Then $\mathcal{H}=L^{2}(X, \mu)$ : To see this, first note that $g \cdot \chi_{K_{n}} \in \mathcal{H}$ for $g \in \mathcal{G}$. Then, if $h \in C(X)$, each $h \cdot \chi_{K_{n}} \in \mathcal{H}$ (since $\varphi$ is bounded on $K_{n}$ ), but this implies that $h \in \mathcal{H}$. Now, use the fact that $C(X)$ is dense in $L^{2}(X)$.

The result will now follow by Lemma 2.1 if we can verify, for each $f \in$ $\mathcal{G}$ and each $m, n, p, q \in \mathbb{N},\left\{g \in \mathcal{G}:\left(g\left(\psi_{n}\right)^{m}, f\left(\psi_{p}\right)^{q}\right)_{\mu} \neq 0\right\}$ is countable. Now for each $r \in \mathbb{N}$, Bessel's Inequality (applied in $L^{2}(X, \nu)$ ) implies that $\int g\left(\psi_{n}\right)^{m} \bar{f}\left(\psi_{p}\right)^{q} \chi_{K_{r}} \varphi d \nu=0$ for all but countably many $g \in \mathcal{G}$, since the function $\left(\psi_{n}\right)^{m} f\left(\psi_{p}\right)^{q} \chi_{K_{r}} \varphi$ is in $L^{2}(X, \nu)$. It follows that $\left(g\left(\psi_{n}\right)^{m}, f\left(\psi_{p}\right)^{q}\right)_{\mu}=$ $\int g\left(\psi_{n}\right)^{m} \bar{f}\left(\psi_{p}\right)^{q} \varphi d \nu=0$ for all but countably many $g \in \mathcal{G}$.

\section{Small Orthogonal Families}

We shall build a large nice $(X, \nu)$ in which every orthogonal family of continuous functions is countable. In order to do this, we apply Lemma 2.4; it is enough to obtain some countable maximal $\mathcal{F} \subseteq C(X)$. Again, we shall, for definiteness, assume that the scalar field is $\mathbb{C}$. $\mathcal{F}$ will be obtained by projecting $X$ onto a small space $M$, for which we use the following notation.

Definition 3.1. $(X, \nu, \Gamma, M)$ is a nice quadruple iff $(X, \nu)$ is a nice measure space and $\Gamma$ is a continuous map onto the compact Hausdorff space $M$. In this case, let $\mu=\nu \Gamma^{-1}$ be the induced measure on $M$. We regard $L^{2}(M, \mu)$ as contained in $L^{2}(X, \nu)$ via the inclusion $\Gamma^{*}\left(\right.$ where $\left.\Gamma^{*}(g)=g \circ \Gamma\right)$. Let $\Pi_{\Gamma}$ 
be the orthogonal projection from $L^{2}(X, \nu)$ onto $L^{2}(M, \mu)$. If $f \in L^{2}(X, \nu)$, we say $f \perp L^{2}(M, \mu)$ iff $\Pi_{\Gamma}(f)=0$.

Lemma 3.2. In the notation of Definition 3.1, if $f \in L^{2}(X, \nu)$ then the following are equivalent:

1. $f \perp L^{2}(M, \mu)$.

2. $\int_{\Gamma^{-1} K} f(x) d \nu(x)=0$ for all closed $K \subseteq M$.

Definition 3.3. The nice quadruple $(X, \nu, \Gamma, M)$ is injective iff $\Pi_{\Gamma}$ is $1-1$ on $C(X)$.

Note that this is the same as saying that a nice quadruple is injective iff for all $f \in C(X)$, if $f \perp L^{2}(M, \mu)$, then $f \equiv 0$.

Lemma 3.4. Let $(X, \nu)$ be a nice measure space. Then the following are equivalent:

1. Every orthogonal subfamily of $C(X)$ is countable.

2. There is a continuous map $\Gamma$ onto a compact second countable space $M$ such that $(X, \nu, \Gamma, M)$ is injective.

Proof. $(2) \rightarrow(1)$ : Assuming $(2)$, let $\mathcal{F} \subseteq C(M)$ be an orthonormal basis for $L^{2}(M)$. Then $\Gamma^{*}(\mathcal{F}) \cup\{0\} \subseteq C(X)$, and is maximal orthogonal, so (1) follows by Lemma 2.4 .

$(1) \rightarrow(2)$ : Again by Lemma 2.4, let $\left\{f_{n}: n \in \mathbb{N}\right\} \subseteq C(X)$ be maximal orthogonal. Let $\Gamma: X \rightarrow \mathbb{C}^{\mathbb{N}}$ be the product map: $(\Gamma(x))_{n}=f_{n}(x)$. Let $M$ be the range of $\Gamma$. Observe that a non-zero $g \in C(X)$ with $\Pi_{\Gamma}(g)=0$ would contradict maximality.

The next lemma explains how we obtain the situation of Lemma 3.4.2:

Lemma 3.5. Let $(X, \nu, \Gamma, M)$ be a nice quadruple. Assume, for some fixed $\epsilon>0$, we have: Whenever $W \subseteq X$ is open and non-empty, there is a closed $K \subseteq M$ such that $\mu(K)>0$ and $\nu\left(\Gamma^{-1}(K) \cap W\right) \geq\left(\frac{1}{2}+\epsilon\right) \mu(K)$. Then $(X, \nu, \Gamma, M)$ is injective.

Proof. Suppose $f \in C(X)$ is non-zero and satisfies $f \perp L^{2}(M, \mu)$. We may assume that $\|f\|_{\text {sup }}=1$, and that some $f(x)=1$. For any $\delta>0$, we may choose a non-empty open $W \subseteq X$ such that $|f(x)-1| \leq \delta$ for all $x \in W$, and then choose $K$ as above. Applying $f \perp L^{2}(M, \mu)$ to the characteristic function of $K$, we have $\int_{\Gamma^{-1} K} f(x) d \nu(x)=0$, so that $\left|\int_{\Gamma^{-1} K \cap W} f\right|=\left|\int_{\Gamma^{-1} K \backslash W} f\right|$. 
Note that $\mu(K)=\nu\left(\Gamma^{-1} K\right)$, so that $\nu\left(\Gamma^{-1} K \backslash W\right) \leq\left(\frac{1}{2}-\epsilon\right) \mu(K)$. So, we have:

$$
\begin{aligned}
& \left|\int_{\Gamma^{-1} K \cap W} f\right| \geq \nu\left(\Gamma^{-1} K \cap W\right)(1-\delta) \geq\left(\frac{1}{2}+\epsilon\right) \mu(K)(1-\delta) \\
& \left|\int_{\Gamma^{-1} K \backslash W} f\right| \leq \nu\left(\Gamma^{-1} K \backslash W\right) \leq\left(\frac{1}{2}-\epsilon\right) \mu(K)
\end{aligned}
$$

So, $\left(\frac{1}{2}+\epsilon\right)(1-\delta) \leq\left(\frac{1}{2}-\epsilon\right)$. Letting $\delta \searrow 0$, we have a contradiction.

Note that if $\epsilon=0$, the lemma could fail; consider $X=M \times 2$, with the product measure.

In general, the Maharam dimension of a measure $\nu$ is the cardinality of an orthonormal basis for $L^{2}(\nu) ; \nu$ is called Maharam-homogeneous iff there is no set $K$ of positive measure such that the dimension of $\nu$ restricted to $K$ is less than the dimension of $\nu$. As usual, $\mathfrak{c}=2^{\aleph_{0}}$.

Theorem 3.6. There is a strictly positive regular Borel probability measure $\nu$ on $2^{\mathfrak{c}}$ (i.e., $\{0,1\}^{\mathfrak{c}}$, with the usual product topology) such that

1. $\nu$ is Maharam-homogeneous of dimension $\mathfrak{c}$.

2. $L^{2}\left(2^{c}, \nu\right)$ contains no uncountable orthogonal family of continuous functions.

Proof. Let $M=2^{\mathbb{N}}$, with $\mu$ the usual product measure. Let $X=M \times 2^{\mathfrak{c}}$, and let $\Gamma: X \rightarrow M$ be projection. We shall build $\nu$ on $X$, which is homeomorphic to $2^{\mathfrak{c}}$.

Let $\left\{d_{m}: m \in \mathbb{N}\right\}$ be dense in $(0,1)^{\mathfrak{c}}$. For each $m$, let $\lambda_{m}$ be the product measure on $2^{\mathfrak{c}}$ obtained by flipping unfair coins with bias $d_{m}$. That is, let $d_{m}^{1}(\alpha)=d_{m}(\alpha)$ and $d_{m}^{0}(\alpha)=1-d_{m}(\alpha)$. If

$$
B=\left\{v \in 2^{\mathfrak{c}}: v\left(\alpha_{1}\right)=\ell_{1} \& \cdots \& v\left(\alpha_{r}\right)=\ell_{r}\right\}
$$

is a basic clopen set, then $\lambda_{m}(B)=\prod_{j=1}^{r} d_{m}^{\ell_{r}}\left(\alpha_{j}\right)$.

List all non-empty clopen subsets of $M$ as $\left\{U_{n}: n \in \mathbb{N}\right\}$. Then, choose closed nowhere dense $K_{m, n} \subseteq U_{n}$ so that the $K_{m, n}$ for $m, n \in \mathbb{N}$ are all disjoint, each $\mu\left(K_{m, n}\right)>0$, and $\sum_{m, n} \mu\left(K_{m, n}\right)=1$. ¿Finally, let $\nu$ on $M \times 2^{\mathfrak{c}}$ be the sum of the product measures $\left(\mu\left\lceil K_{m, n}\right) \times \lambda_{m}\right.$, so that for Borel $E \subseteq M \times 2^{\mathfrak{c}}$,

$$
\nu(E)=\sum_{m, n} \int_{K_{m, n}} \lambda_{m}\left(E_{x}\right) d \mu(x) .
$$

We are now done if we can verify the hypotheses of Lemma 3.5 We actually show that whenever $W \subseteq X$ is open and non-empty and $\epsilon>0$, there is a closed 
$K \subseteq M$ such that $\mu(K)>0$ and $\nu\left(\Gamma^{-1}(K) \cap W\right) \geq(1-\epsilon) \mu(K)$. To do this, we may assume that $W=U_{n} \times B$, where $B$ is as in (1) above. $K$ will be $K_{m, n}$ for a suitable $m$. Then $\nu\left(\Gamma^{-1}(K) \cap W\right)=\nu\left(K_{m, n} \times B\right)=\mu\left(K_{m, n}\right) \prod_{j=1}^{r} d_{m}^{\ell_{r}}\left(\alpha_{j}\right)$. We thus only need choose $m$ so that $\prod_{j=1}^{r} d_{m}^{\ell_{r}}\left(\alpha_{j}\right) \geq(1-\epsilon)$, which is certainly possible since $\left\{d_{m}: m \in \mathbb{N}\right\}$ is dense in $(0,1)^{\mathfrak{c}}$.

Finally, we remark that this example is as large as possible, since whenever $|C(X)|>\mathfrak{c}$, there is an uncountable orthogonal family, by Lemma 3.4. (Note that if $X$ is an infinite compact Hausdorff space, then $|C(X)|=w(X)^{\aleph_{0}}$, where $w(X)$ is the weight of $X$ (the least size of a base for the topology)). One can, however, construct arbitrarily large examples with no continuous orthonormal bases by applying:

Theorem 3.7. Suppose that $(X, \nu)$ and $(Y, \rho)$ are both nice measure spaces, and there is an orthonormal basis for $L^{2}(X \times Y, \nu \times \rho)$ contained in $C(X \times$ $Y)$. Then there are orthonormal bases for $L^{2}(X, \nu), L^{2}(Y, \rho)$ contained in $C(X), C(Y)$, respectively.

Proof. Let $\mathcal{G} \subseteq C(X \times Y)$ be an orthonormal basis for $L^{2}(X \times Y, \nu \times \rho)$. To produce a basis for $L^{2}(X, \nu)$, let $\Gamma: X \times Y \rightarrow X$ be projection, and apply Lemma 2.1, with $\mathcal{E}=\Pi_{\Gamma}(\mathcal{G}) \subseteq \mathcal{H}=L^{2}(X, \nu)$ (regarding $L^{2}(X)$ as contained in $L^{2}(X \times Y)$, as in Definition 3.1).

First, note that the closed linear span of $\mathcal{E}$ will be all of $L^{2}(X)$, because the closed linear span of $\mathcal{G}$ is $L^{2}(X \times Y)$ and $\Pi_{\Gamma}$ is orthogonal projection.

Next, observe that for each $G \in \mathcal{G}, \Pi_{\Gamma}(G)=g$, where $g(x)=\int G(x, y) d y$. To see this, note that since $G$ is continuous, $g \in C(X) \subseteq L^{2}(X)$. Also, for each $f \in L^{2}(X)$,

$$
(g, f)=\int g(x) \bar{f}(x) d x=\iint G(x, y) \bar{f}(x) d x d y=(G, f)
$$

So $\Pi_{\Gamma}(G)=g$ follows from the uniqueness of orthogonal projections.

In particular, $\mathcal{E} \subseteq C(X)$, so that Lemma 2.1 will produce an orthonormal base contained in $C(X)$.

Finally, countability of $\mathcal{E}_{f}=\{g \in \mathcal{E}:(g, f) \neq 0\}$, for any $f \in \mathcal{E}$, follows from Bessel's inequality: For each $g=\Pi_{\Gamma}(G) \in \mathcal{E}$, since $(g, f)=(G, f)$, we have $\sum\left\{|(G, f)|^{2}: G \in \mathcal{G}\right\} \leq\|f\|^{2}$.

For example, let $\kappa$ be any infinite cardinal such that $\kappa^{\aleph_{0}}=\kappa$. We may then obtain a nice $(Z, \mu)$ such that $|C(Z)|=\kappa$ and there is no orthonormal basis for $L^{2}(Z, \mu)$ contained in $C(Z)$; we just start with an $X$ as in Theorem 3.6, and then $Z=X \times Y$ for a suitable $Y$ (applying Theorem 3.7). However, 
assuming also that $2^{\lambda}<\kappa$ for all $\lambda<\kappa$ (for example, $\kappa$ could be $\beth_{\omega_{1}}$, or $\kappa$ could be strongly inaccessible), every maximal orthogonal family $\mathcal{F} \subseteq C(Z)$ must have size $\kappa$ : If $|\mathcal{F}|=\lambda<\kappa$, we could always find distinct $g, h \in C(Z)$ such that $(g, f)=(h, f)$ for all $f \in \mathcal{F}$ (since there are only $2^{\lambda}<\kappa=|C(Z)|$ possibilities for $\langle(g, f): f \in \mathcal{F}\rangle)$. Then $(g-h) \perp \mathcal{F}$, so $\mathcal{F}$ cannot be maximal.

\section{References}

[1] G. B. Folland, A Course in Abstract Harmonic Analysis, CRC Press, 1995. 